\section{Dynamic Assessment}

Philip Schatz

Department of Psychology, Saint Joseph's

University, Philadelphia, PA, USA

\section{Definition}

Dynamic assessment refers to an interactive approach to conducting assessments, focusing on the ability of the examinee to respond to an intervention. As an alternative to a more traditional assessment approach, within dynamic assessment, the evaluator conducts a pretest, imposes an intervention, and then conducts a posttest. Comparison of changes from pre- to posttest performance allows the evaluator to determine the examinee's potential for learning.

\section{References and Readings}

Haywood, H. C., \& Lidz, C. S. (2006). Dynamic assessment in practice: Clinical and educational applications. New York: Cambridge University Press. 